\title{
DUSP4 promotes doxorubicin resistance in gastric cancer through epithelial-mesenchymal transition
}

\author{
Xing Kang ${ }^{1, *}$, Minhuan Li ${ }^{2, *}$, Hao Zhu ${ }^{3, *}$, Xiaofeng Lu ${ }^{1}$, Ji Miao ${ }^{1}$, Shangce Du ${ }^{1}$, Xuefeng \\ $\mathrm{Xia}^{1}$ and Wenxian Guan ${ }^{1}$ \\ ${ }^{1}$ Department of General Surgery, The Affiliated Drum Tower Hospital of Nanjing University Medical School, Nanjing 210008, \\ Jiangsu Province, China \\ ${ }^{2}$ Department of Laboratory Medicine, Sir Run Run Hospital, Nanjing Medical University, Nanjing, 210000, Jiangsu Province, \\ China \\ ${ }^{3}$ Department of Gastroenterology, The Afflicted Drum Tower Hospital, Nanjing University Medical School, Nanjing 210008, \\ Jiangsu Province, China \\ *These authors have contributed equally to this work \\ Correspondence to: Xuefeng Xia, email: $18114483312 @$ sina.cn \\ Wenxian Guan, email: 15850502391@163.com
}

Keywords: chemoresistance, doxorubicin, DUSP4, epithelial-mesenchymal transition, gastric cancer

Received: August 08, $2017 \quad$ Accepted: September 03, $2017 \quad$ Published: October 04, 2017

Copyright: Kang et al. This is an open-access article distributed under the terms of the Creative Commons Attribution License 3.0 (CC BY 3.0), which permits unrestricted use, distribution, and reproduction in any medium, provided the original author and source are credited.

\section{ABSTRACT}

Chemoresistance limits treatment efficacy in gastric cancer and doxorubicin resistance is common in gastric cancer cells. Dual specificity phosphatase 4 (DUSP4) has been associated with tumor progression. This study aimed to investigate the mechanism of DUSP4 regulating doxorubicin resistance in gastric cancer cells. Cell Counting Kit-8 (CCK-8) and 5-ethynyl-2'-deoxyuridine (EdU) incorporation assay were used to measure cell viability and proliferation in gastric cancer cells treated with doxorubicin. The expression of DUSP4, E-cadherin and Vimentin protein was detected by Western blotting. Overexpression of DUSP4 was more resistant to doxorubicin in gastric cancer cells. Knockdown of DUSP4 increased the sensitivity of gastric cancer cells to doxorubicin. Moreover, up-regulation of DUSP4 promoted the EpithelialMesenchymal Transition (EMT) in gastric cancer cells, but blocking the EMT using a Twist siRNA increased the sensitivity of gastric cancer cells to doxorubicin and confirmed the EMT was involved in DUSP4-mediated doxorubicin resistance. These findings demonstrated that DUSP4 could enhance doxorubicin resistance by promoting EMT in gastric cancer cells.

\section{INTRODUCTION}

Gastric cancer (GC) is one of the most common digestive system malignancies and has a high mortality rate, accounting for approximately $9 \%$ of cancer deaths worldwide [1]. Most patients with newly diagnosed GC have advanced stage disease that is not resectable [2, 3]. Moreover, patients who undergo complete tumor resection have a high risk of recurrence [4]. Chemotherapy has been widely adopted to prolong survival in patients with advanced GC. However, the overall survival rate of advanced GC remains unsatisfactory due to the development of multidrug resistance $[5,6]$. Therefore, the mechanisms underlying chemotherapy resistance in GC are of major interest.

Doxorubicin (DOX) is an anthracycline-based chemotherapeutic agent that has been used as the gold-standard therapy for advanced GC since 1980 [7]. However, DOX-based regimens are not currently recommended as a first-line chemotherapy strategy for $\mathrm{GC}$ due to frequent development of resistance and poor efficacy. The epithelial-to-mesenchymal transition (EMT) is a major mechanism associated with drug resistance. The EMT has been reported to promote cancer cell metastasis and resistance to chemotherapy $[8,9]$. Recent 
studies demonstrated that the frequent acquisition of DOX resistance in cancer may be related to the EMT [10-14]. The protein alterations that occur during the EMT include a loss of epithelial markers, such as E-cadherin, and acquisition of mesenchymal markers, such as vimentin [15]. Several studies have investigated the relationship between the EMT and DOX-resistance in GC, and many factors involved in EMT mediated DOX-resistance have been discovered [16, 17]. However, the precise mechanisms by which the EMT is associated with DOXresistance have not yet been fully uncovered.

Dual specificity phosphatase 4 (DUSP4), a member of the DUSP family, is also known as MAPK phosphatase 2 (MKP2). DUSP4 plays an essential role in regulation of cell proliferation and differentiation via interacting with the MAPK signaling pathway [18]. However, contradictory roles have been reported for DUSP4 in cancer. As a negative regulator of the MAPK signaling pathway, DUSP4 may represent as a tumor suppressor gene. Downregulation of DUSP4 is associated with progression in several types of cancer, including colorectal cancer [19], breast cancer [20], pancreatic cancer [21] and diffuse large B cell lymphoma [22]. However, other studies indicate that overexpression of DUSP4 promotes cancer development and progression [23-25]. Interestingly, DUSP4 has been related to drug resistance in several cancers [26-30]. However, little is known about the role of DUSP4 in DOX resistance in GC.

In the present study, we aimed to explore the role of DUSP4 in DOX resistance in GC cell lines, uncover the associated mechanisms, and investigate the link between the EMT and doxorubicin resistance in gastric cancer.

\section{RESULTS}

\section{GC cells expressing low levels of DUSP4 are more sensitive to $\mathrm{DOX}$}

To explore the effect of DUSP4 on the sensitivity of GC cells to DOX, the expression of DUSP4 was measured by Western blotting. KATOIII and MKN45 cells expressed high levels of DUSP4 compared to BGC and SGC7901 cells (Figure 1A). Next, we measured cell
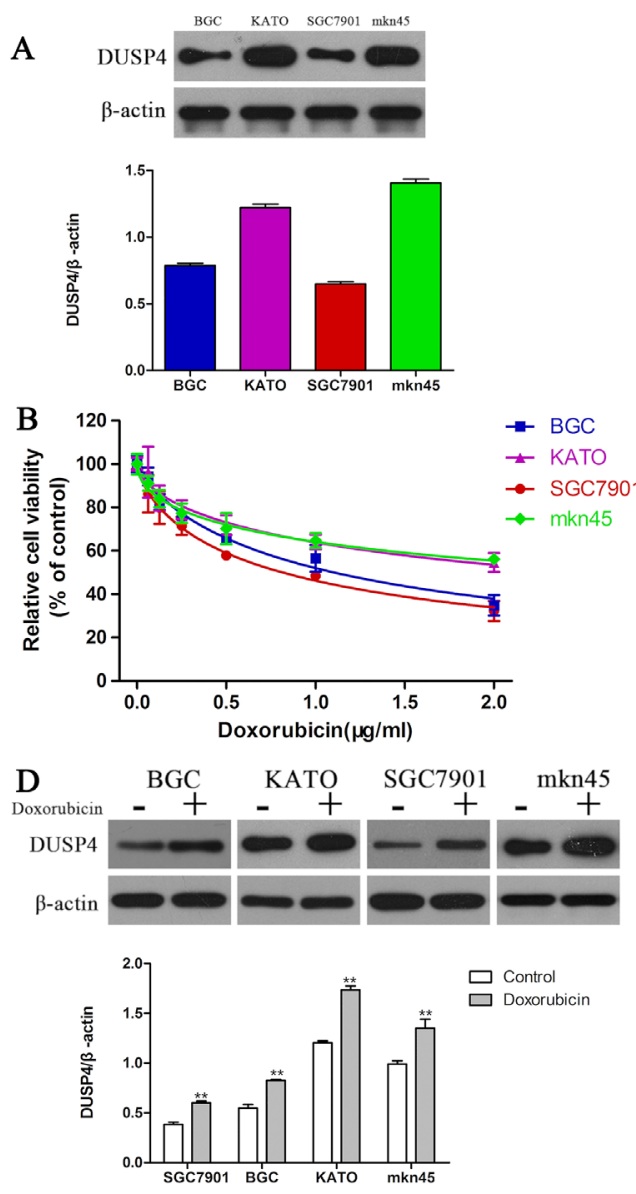

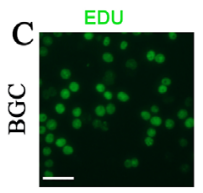

Hoechest 3344
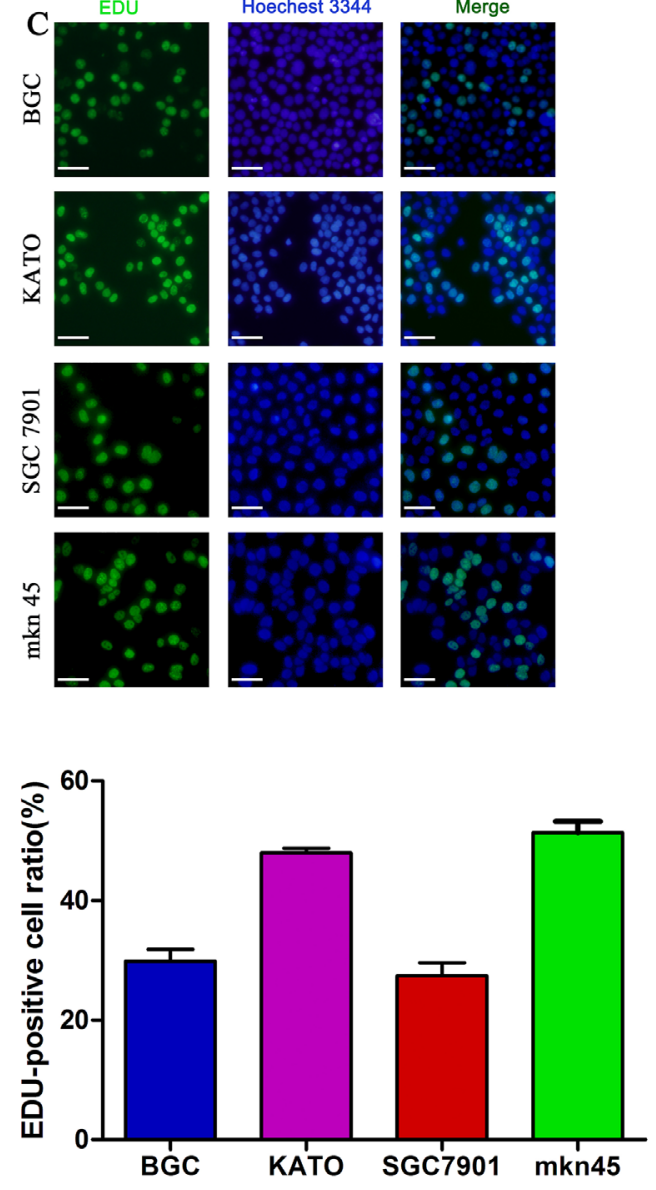

Figure 1: Expression of DUSP4 is associated with DOX resistance in GC cells. (A) Western blot of DUSP4 protein expression in GC cells. (B) CCK-8 assay of the viability of GC cells treated with different concentrations of DOX. (C) EDU assay of the proliferation of GC cells treated with their $\mathrm{IC}_{50}$ of DOX. (D) Western blot of DUSP4 protein expression in $\mathrm{GC}$ cells cultured with or without their $\mathrm{IC}_{50}$ of DOX. $\left({ }^{* *} P<0.01\right)$. 
Table 1: The viability of GC cells treated with different concentrations of DOX

\begin{tabular}{lcccc}
\hline cell lines & SGC7901 & BGC & KATO & mkn45 \\
\hline $\mathrm{IC}_{50}(\mu \mathrm{g} / \mathrm{ml})$ & $0.8146(0.7179$ to 0.9112$)$ & $1.103(0.9786$ to 1.227$)$ & $2.507(1.760$ to 3.254$)$ & $3.025(2.205$ to 3.845$)$ \\
\hline
\end{tabular}

$\mathrm{IC}_{50}$ values show Doxorubicin concentration $[\mu \mathrm{g} / \mathrm{ml}$. mean (95\% confidence intervals) $]$.

viability after $48 \mathrm{~h}$ DOX treatment using the CCK-8 assay. All four GC cells exhibited dose-dependent reductions in cell viability in response to DOX (Figure 1B, Table 1). However, DUSP4 ${ }^{\text {high }}$ KATO III and MKN45 cells were more resistant to DOX than DUSP4 ${ }^{\text {low }}$ BGC and SGC7901 cells. The EDU cell proliferation assay confirmed these results (Figure 1C). The $\mathrm{IC}_{50}$ values of DOX in KATOIII, MKN45, BGC and SGC7901 cells at $48 \mathrm{~h}$ were 2.507, $3.025,1.103$ and $0.8146 \mu \mathrm{g} / \mathrm{mL}$, respectively (Table 1 ). Moreover, treatment with DOX at the $\mathrm{IC}_{50}$ values for $48 \mathrm{~h}$ obviously increased the expression of DUSP4 in all four GC cell lines (Figure 1D) $\left({ }^{* *} P<0.01\right)$. Taken together, we deduced that DUSP4 may mediate DOX resistance in GC cells.

\section{DUSP4 promotes DOX resistance in GC cells}

To further explore the relationship between DUSP4 and DOX resistance in GC cells, we employed a siRNA to knockdown DUSP4 and a plasmid to overexpress DUSP4. The efficacy of the siRNA and plasmid were confirmed by Western blotting (Figure $2 \mathrm{~A})\left({ }^{*} P<0.05,{ }^{* * *} P<\right.$ $0.001)$. The CCK-8 assay revealed knockdown of DUSP4 significantly enhanced the cytotoxicity of DOX in GC cells. Conversely, overexpression of DUSP4 significantly reduced the cytotoxicity of DOX (Figure 2B-2E, Table 2). The EDU cell proliferation assay confirmed the results of the CCK-8 assay (Figure 2F) $\left({ }^{*} P<0.05,{ }^{* *} P<0.01,{ }^{* * *} P\right.$ $<0.001)$. These data confirmed that DUSP4 significantly enhances the resistance of GC cells to DOX.

\section{The EMT underlies DOX resistance in GC cells}

As the EMT plays a role in chemoresistance in several solid tumors, we hypothesized the EMT may mediate DOX resistance in GC cells. To prove this hypothesis, we first investigated the expression of EMT marker proteins in the four GC cell lines by Western blotting. DOX-resistant DUSP4 $4^{\text {high }}$ KATOIII and MKN45 cells expressed low levels of E-cadherin and high levels of vimentin, in contrast to DOX-sensitive DUSP4 ${ }^{\text {low }}$ BGC and SGC7901 cells (Figure 3A). Treatment with
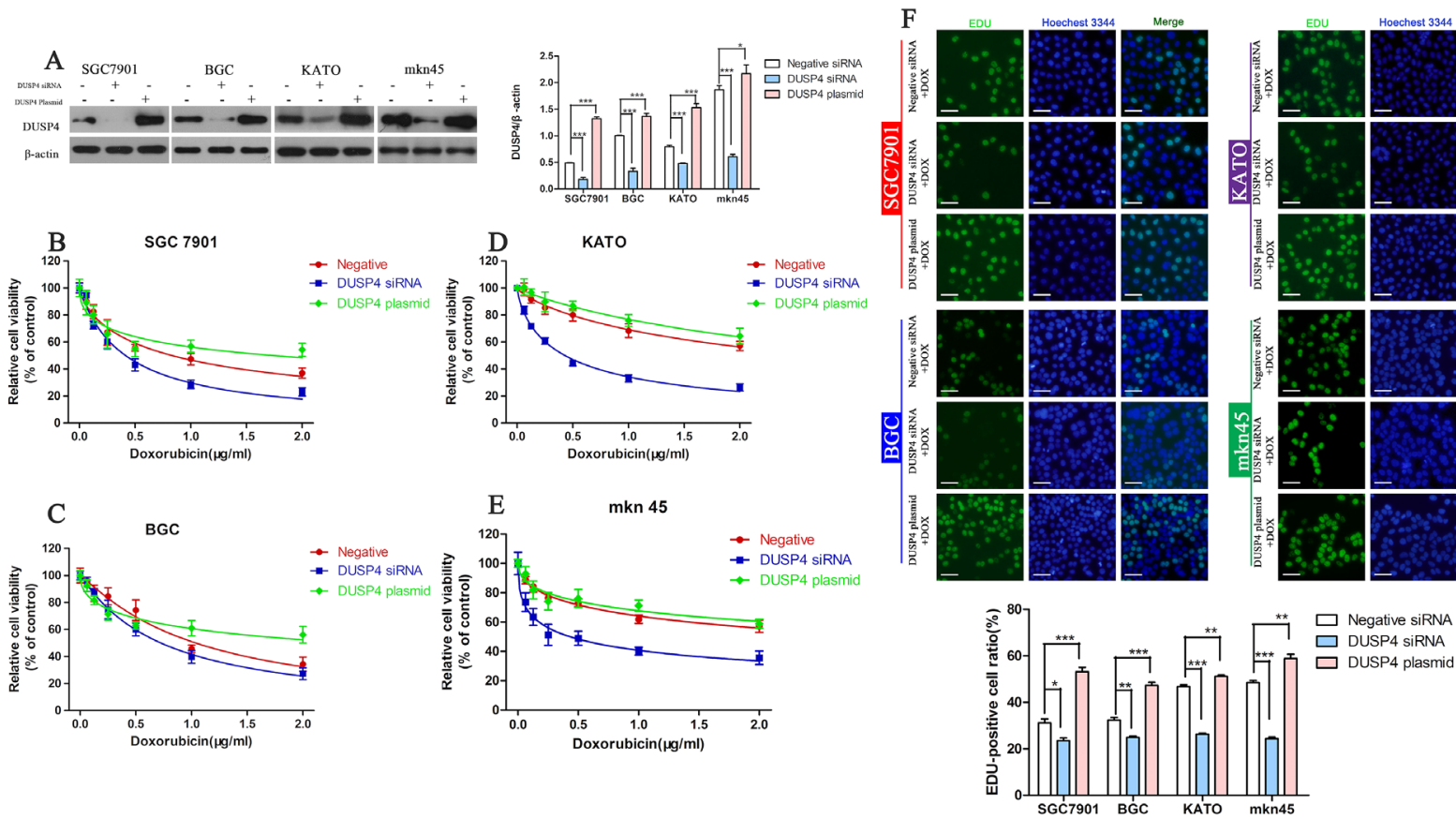

Figure 2: Knockdown of DUSP4 increases the sensitivity of GC cells to DOX. (A) Western blot confirmation of the efficiency of DUSP4 knockdown or overexpression; $\beta$-actin was used as the internal control $\left({ }^{*} P<0.05,{ }^{* * *} P<0.001\right)$. (B-E) CCK-8 assay of the viability of GC cells in which DUSP4 was knocked down or overexpressed treated with different concentrations of DOX. (F) EDU assay of the proliferation of GC cells in which DUSP4 was knocked down or overexpressed treated with their $\mathrm{IC}_{50}$ of DOX. $\left({ }^{*} P<0.05\right.$, ${ }^{* *} P<0.01$, $\left.{ }^{* * *} P<0.001\right)$. 
Table 2: The viability of GC cells in which DUSP4 was knocked down or overexpressed treated with different concentrations of DOX

\begin{tabular}{lccc}
\hline \multirow{2}{*}{ cell lines } & \multicolumn{3}{c}{$\mathbf{I C}_{\mathbf{5 0}}(\boldsymbol{\mu g} / \mathbf{m l})$} \\
\cline { 2 - 4 } & Negative+ DOX & DUSP4 siRNA+ DOX & DUSP4 plasmid+DOX \\
\hline SGC7901 & $0.831(0.7142$ to 0.9478$)$ & $0.4135(0.3733$ to 0.4538$)$ & $1.734(0.9695$ to 2.498$)$ \\
BGC & $1.039(0.9223$ to 1.155$)$ & $0.7258(0.6675$ to 0.7841$)$ & $2.426(1.624$ to 3.228$)$ \\
KATO & $2.755(2.262$ to 3.247$)$ & $0.4289(0.4031$ to 0.4547$)$ & $3.718(2.742$ to 4.694$)$ \\
mkn45 & $3.198(2.536$ to 3.860$)$ & $0.4323(0.3490$ to 0.5155$)$ & $5.127(2.440$ to 7.814$)$ \\
\hline
\end{tabular}

$\mathrm{IC}_{50}$ values show Doxorubicin concentration $[\mu \mathrm{g} / \mathrm{ml}$. mean $(95 \%$ confidence intervals $)]$.

the $\mathrm{IC}_{50}$ of DOX for $48 \mathrm{~h}$ increased vimentin expression and decreased E-cadherin expression compared to the respective untreated cells (Figure 3B) $\left({ }^{* * *} P<0.01,{ }^{* * *} P<\right.$ 0.001 ), indicating DOX can induce the EMT in GC cells.

Twist is an essential regulator of the EMT in cancer cells [31]. Therefore, we used a siRNA to knockdown Twist to inhibit the EMT. The CCK-8 assay revealed that knockdown of Twist increased the sensitivity of all four GC cell lines to DOX (Figure 3C-3F, Table 3) and the EDU assay confirmed these results (Figure $3 \mathrm{G})\left({ }^{* *} P\right.$ $<0.01,{ }^{* * *} P<0.001$ ), strongly suggesting that the EMT contributes to DOX resistance in GC cells.
Hypoxic culture can induce the EMT in cancer cell lines $[32,33]$. To further confirm the role of the EMT in DOX resistance, we examined the viability and proliferation of GC cells cultured under hypoxic and normoxic conditions. Our results showed that DOX could reduce the viability of all four GC cell lines in a dosedependent manner; however, cells cultured under hypoxic conditions were more resistant to DOX than those cultured under normoxic conditions (Figure 4A-4D, Table 4). The EDU assay confirmed hypoxia increased cell proliferation in a similar manner in DOX treated cells (Figure 4E) $\left({ }^{*} P<\right.$
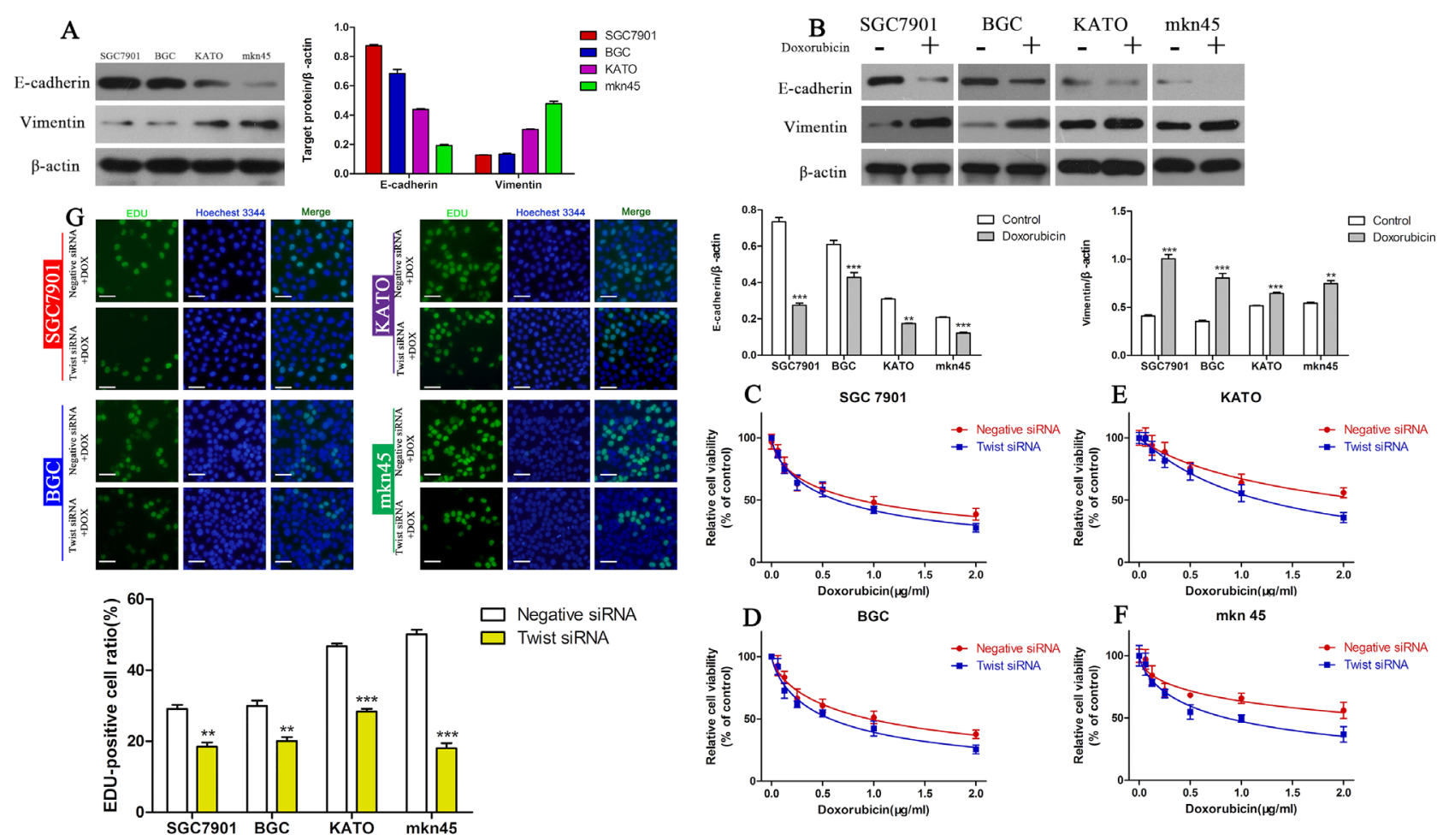

Figure 3: The EMT mediates DOX resistance in GC cell lines. (A) Western blots of E-cadherin and vimentin protein expression in the four $\mathrm{GC}$ cell lines. (B) Western blots of E-cadherin and vimentin protein expression in the four $\mathrm{GC}$ cell lines cultured with their $\mathrm{IC}_{50}$ of DOX $\left({ }^{* *} P<0.01,{ }^{* * *} P<0.001\right)$. (C-F) CCK-8 assay of the viability of GC cells in which Twist was knocked down cultured in different concentrations of DOX. (G) EDU assay of the proliferation of GC cells in which Twist was knocked down cultured in their $\mathrm{IC}_{50}$ of DOX $\left({ }^{* *} P<0.01,{ }^{* * *} P<0.001\right)$. 
Table 3: The viability of GC cells in which Twist was knocked down cultured in different concentrations of DOX

\begin{tabular}{lcc}
\hline \multirow{2}{*}{ cell lines } & \multicolumn{2}{c}{$\mathbf{I C}_{\mathbf{5 0}}(\boldsymbol{\mu g} / \mathbf{m l})$} \\
\cline { 2 - 3 } & Negative siRNA+ DOX & Twist siRNA+ DOX \\
\hline SGC7901 & $0.8609(0.7169$ to 1.005$)$ & $0.6367(0.5718$ to 0.7017$)$ \\
BGC & $0.9592(0.8089$ to 1.110$)$ & $0.5751(0.5060$ to 0.6443$)$ \\
KATO & $2.267(1.726$ to 2.807$)$ & $1.195(1.057$ to 1.334$)$ \\
mkn45 & $2.765(1.650$ to 3.879$)$ & $0.8573(0.7188$ to 0.9957$)$ \\
\hline
\end{tabular}

$\mathrm{IC}_{50}$ values show Doxorubicin concentration $[\mu \mathrm{g} / \mathrm{ml}$. mean ( $95 \%$ confidence intervals)].

$\left.0.05,{ }^{* *} P<0.01,{ }^{* * *} P<0.001\right)$. These findings prove the

EMT promotes DOX resistance in GC cells.

\section{Knockdown of DUSP4 inhibits the EMT in GC cells}

Next, we investigated whether DUSP4 is involved in the EMT in GC cells. We transfected the DUSP4
siRNA or DUSP4 plasmid into the four GC cell lines, and quantified the expression of E-cadherin and vimentin. Knockdown of DUSP4 increased E-cadherin expression and reduced vimentin expression (Figure 5A). In contrast, overexpression of DUSP4 had the opposite effects (Figure 5A) $\left({ }^{*} P<0.05,{ }^{* *} P<0.01,{ }^{* * *} P<0.001\right)$. These results indicated DUSP4 may regulate the EMT in GC cells.
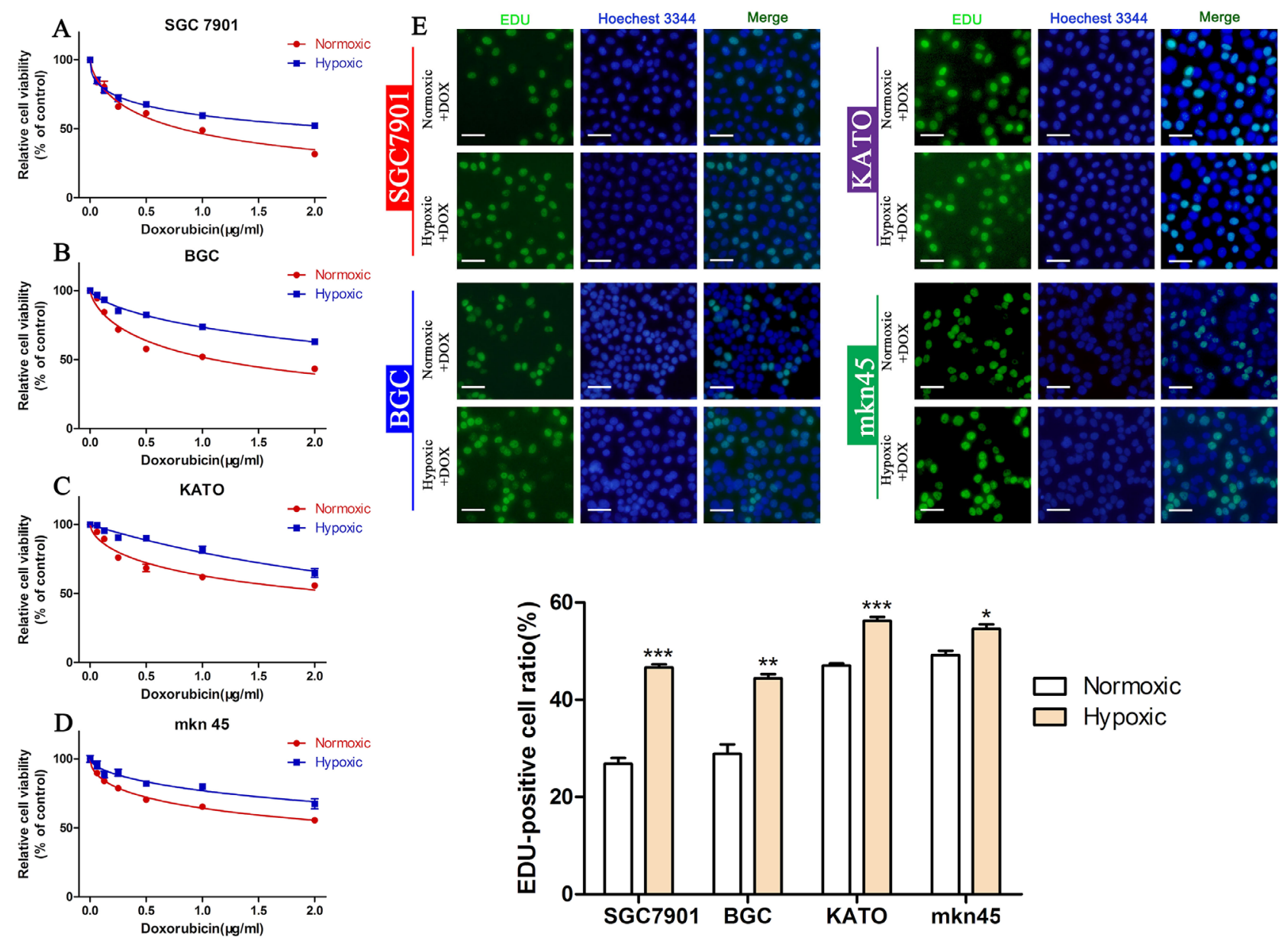

Figure 4: Hypoxia induces DOX resistance in GC cell lines. (A-D) CCK-8 assay of the viability of GC cells treated with different concentrations of DOX cultured under hypoxic or normoxic conditions. (E) EDU assay of the proliferation of GC cells treated with different concentrations of DOX cultured under hypoxic or normoxic conditions $\left({ }^{*} P<0.05,{ }^{* *} P<0.01,{ }^{* * *} P<0.001\right)$. 
Table 4: The viability of GC cells treated with different concentrations of DOX cultured under hypoxic or normoxic conditions

\begin{tabular}{lcc}
\hline \multirow{2}{*}{ cell lines } & \multicolumn{2}{c}{$\mathbf{I C}_{50}(\boldsymbol{\mu g} / \mathbf{m l})$} \\
\cline { 2 - 3 } & Normoxic+ DOX & Hypoxic+ DOX \\
\hline SGC7901 & $0.8109(0.6978$ to 0.9241$)$ & $2.429(1.813$ to 3.045) \\
BGC & $1.104(0.9518$ to 1.256$)$ & $4.186(3.451$ to 4.921$)$ \\
KATO & $2.365(1.759$ to 2.971$)$ & $3.891(2.792$ to 4.991$)$ \\
mkn45 & $3.052(2.339$ to 3.766$)$ & $7.746(2.586$ to 12.91$)$ \\
\hline
\end{tabular}

$\mathrm{IC}_{50}$ values show Doxorubicin concentration $[\mu \mathrm{g} / \mathrm{ml}$. mean $(95 \%$ confidence intervals $)]$.

\section{DUSP4 mediates DOX sensitivity in GC cells by regulating the EMT}

Based on the results above, we inferred DUSP4 promotes DOX resistance in GC cells by regulating the EMT. To prove this, we examined the cell viability of cells overexpressing DUSP4, transfected with the Twist siRNA to inhibit the EMT and treated with DOX. Overexpression of DUSP4 did not further enhance DOX resistance when the EMT was blocked using the Twist siRNA (Figure 6A-6D, Table 5). The EDU assay confirmed these results (Figure 6E).

As shown in Figure 4, hypoxia could induce the EMT and enhance DOX resistance in GC cells. We investigated whether knockdown of DUSP4 could reverse the hypoxia-induced EMT and hypoxia-mediated DOX resistance. Cell viability and proliferation assays revealed that knockdown of DUSP4 could alleviate hypoxiamediated DOX resistance in GC cells (Figure 7A-7E, Table 6) $\left({ }^{* * *} P<0.001\right)$. These data demonstrate that DUSP4 enhances DOX resistance by regulating the EMT in GC cells.

\section{DISCUSSION}

Chemotherapy is the most effective treatment for advanced GC [3]. However, drug resistance limits the application of conventional DOX-based chemotherapy
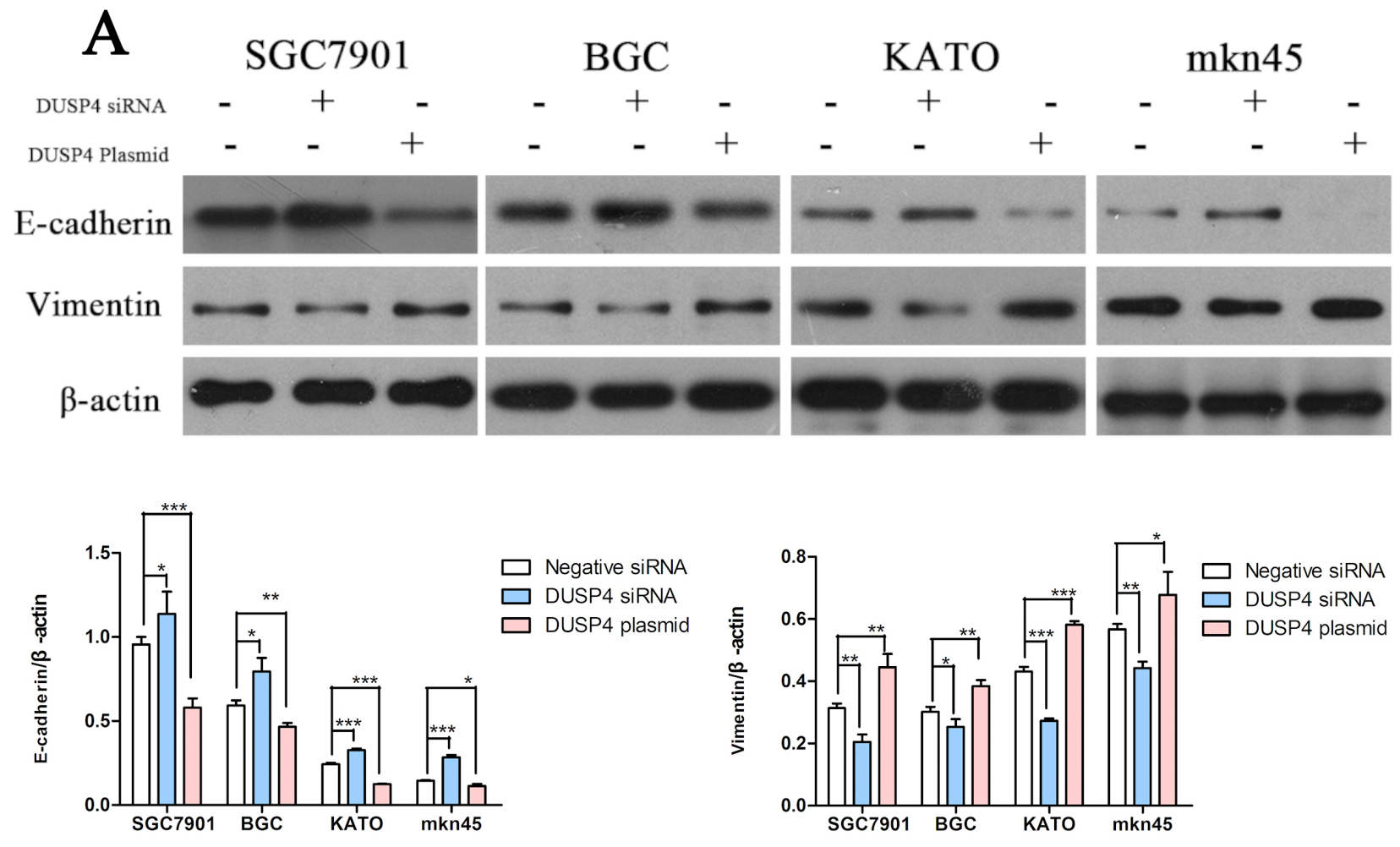

Figure 5: DUSP4 can induce the epithelial-mesenchymal transition (EMT) in GC cell lines. Western blots of E-cadherin and vimentin protein expression in GC cells in which DUSP4 was knocked down or overexpressed $\left({ }^{* *} P<0.05,{ }^{* *} P<0.01,{ }^{* * *} P<0.001\right)$. 
regimens in GC [34]. Thus, it is essential to explore the mediators and mechanisms of drug resistance in GC. In this study, we showed that overexpression of DUSP4 enhanced DOX resistance in GC cell lines by regulating the EMT. To the best of our knowledge, this is the first report of a relationship between DUSP4 and DOX resistance in $\mathrm{GC}$.

The dual specificity phosphatase DUSP4 specifically inactivates MAPK pathway kinases [18]. However, the role of DUSP4 in cancer remains controversial. Some studies have demonstrated DUSP4 is overexpressed and could promote progression in a number of cancers, including colorectal [19] and breast cancer [30, 35]. Conversely, other studies indicated DUSP4 is downregulated and inhibits tumor development in colorectal cancer [3638] and breast cancer [25]. However, few studies have quantified DUSP4 expression in GC. One study reported that decreased DUSP4 expression was associated with sex, tumor size, depth of invasion and distant metastasis in human GC, and that overexpression of DUSP4 reduced GC cell viability and invasive potential and induced cell apoptosis and S phase cycle arrest [39]. However, little is known about the role of DUSP4 in chemoresistance in GC. The present study demonstrates DUSP4 promotes $\mathrm{DOX}$ resistance in $\mathrm{GC}$, in agreement with findings in breast cancer [28].

The EMT confers increased invasion and migration ability and is associated with drug resistance [8, 9]. EMTinduced stemness may represent one potential mechanism underlying EMT-mediated chemoresistance [40]. A previous study showed that long-term, incremental DOX treatment led to drug resistance and the EMT in SGC7901/ Dox cells [41]. Moreover, treatment with DOX for $48 \mathrm{~h}$ induced the EMT in human GC BGC-823 cells, and inhibition of $\beta$-catenin signaling suppressed the DOXinduced EMT and cell migration [16]. In agreement with these previous studies, we found the GC cell lines with a more mesenchymal phenotype, characterized by high vimentin and low E-cadherin expression, were more resistant to DOX. Furthermore, DOX induced vimentin and inhibited E-cadherin expression, indicting
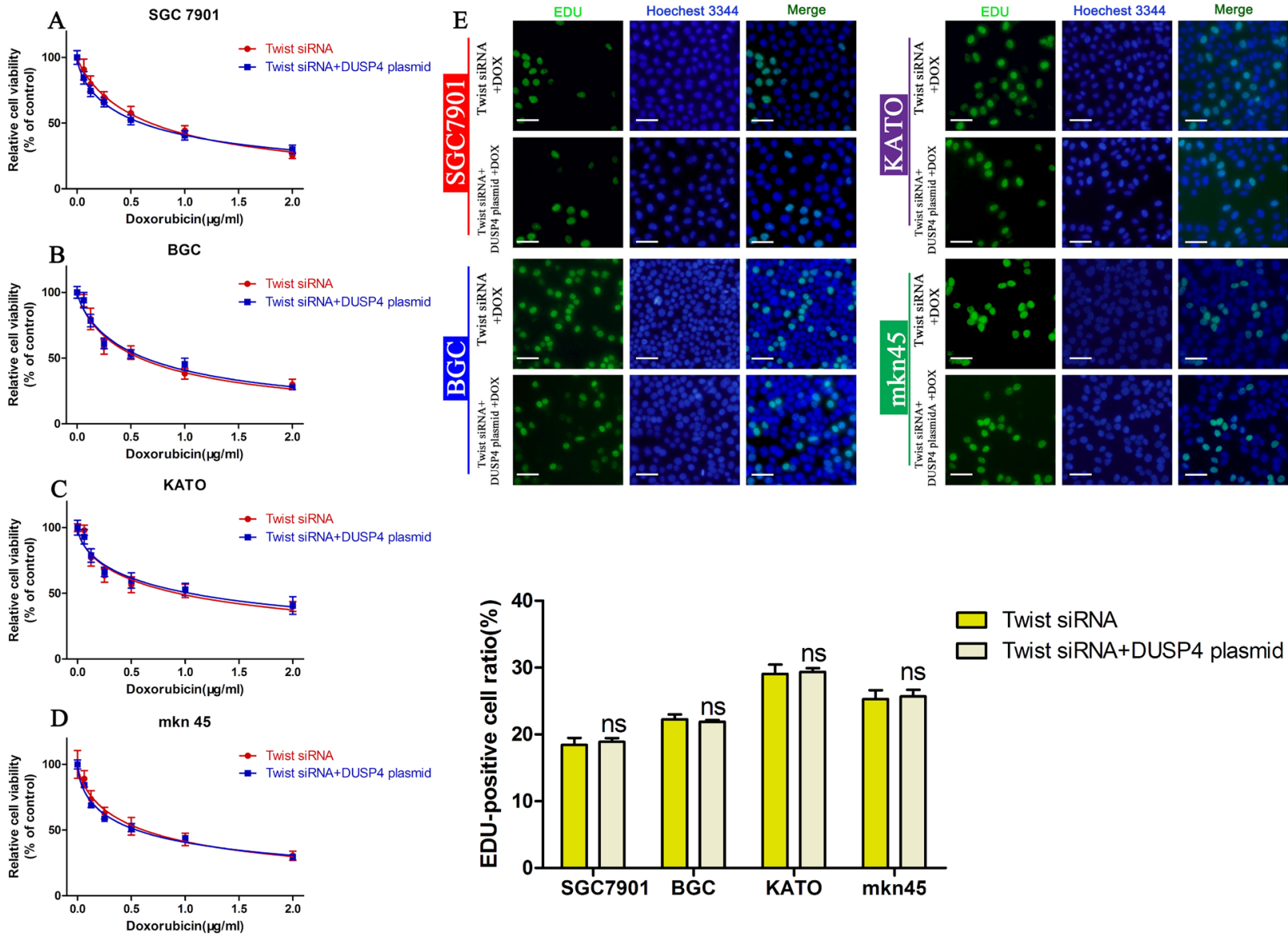

Figure 6: Blocking the EMT attenuates the ability of DUSP4 overexpression to induce DOX resistance. (A-D) CCK-8 assay of the viability of GC cells transfected with the Twist siRNA or Twist siRNA + DUSP4 overexpressing plasmid treated with different concentrations of DOX. (E) EDU assay of the proliferation of GC cells transfected with the Twist siRNA or Twist siRNA + DUSP4 plasmid treated with their $\mathrm{IC}_{50}$ of DOX (NS: not significant). 
Table 5: The viability of GC cells transfected with the Twist siRNA or Twist siRNA + DUSP4 overexpressing plasmid treated with different concentrations of DOX

\begin{tabular}{lcc}
\hline \multirow{2}{*}{ cell lines } & \multicolumn{2}{c}{$\mathbf{I C}_{50}(\boldsymbol{\mu g} / \mathbf{m l})$} \\
\cline { 2 - 3 } & Twist siRNA+ DOX & TwistsiRNA+DUSP4 plasmid + DOX \\
\hline SGC7901 & $0.6835(0.6131$ to 0.7540$)$ & $0.5982(0.5448$ to 0.6515$)$ \\
BGC & $0.5835(0.5047$ to 0.6622$)$ & $0.6311(0.5534$ to 0.7088$)$ \\
KATO & $0.9308(0.7426$ to 1.119$)$ & $1.042(0.8521$ to 1.233$)$ \\
mkn45 & $0.6126(0.5211$ to 0.7040$)$ & $0.5475(0.4976$ to 0.5974$)$ \\
\hline
\end{tabular}

values show Doxorubicin concentration $[\mu \mathrm{g} / \mathrm{ml}$. mean (95\% confidence intervals)].

DOX induced the EMT in all four GC cell lines, and we confirmed the EMT mediates DOX resistance in GC.

Only two studies have reported a relationship between DUSP4 and the EMT. Boulding et al. demonstrated that DUSP1, DUSP4 and DUSP6 are induced during the EMT in breast cancer, and knockdown of DUSP4 could enhance breast cancer stem celldefine (CSC) formation [42]. Liu Y et al. found that overexpression of DUSP4 may promote the EMT in breast cancer, whereas knockdown of DUSP4 enhanced the sensitivity of chemotherapeutic agents in breast cancer cells [28]. Similarly, this study provides the first proof that DUSP4 can induce the EMT in GC and that blocking the EMT could reverse DUSP4-mediated DOX resistance in GC.
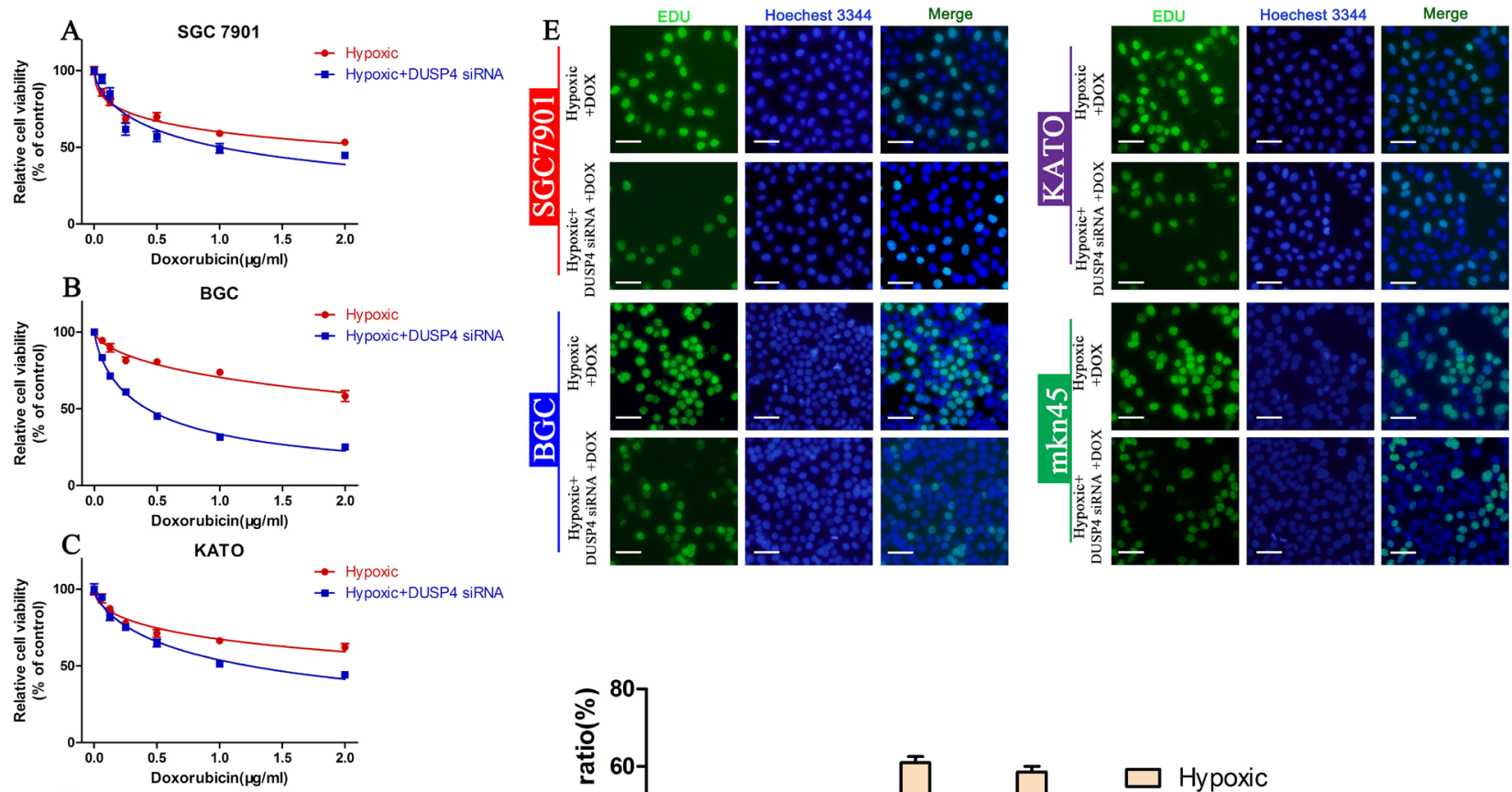

- HypoxictDusp4 sirva
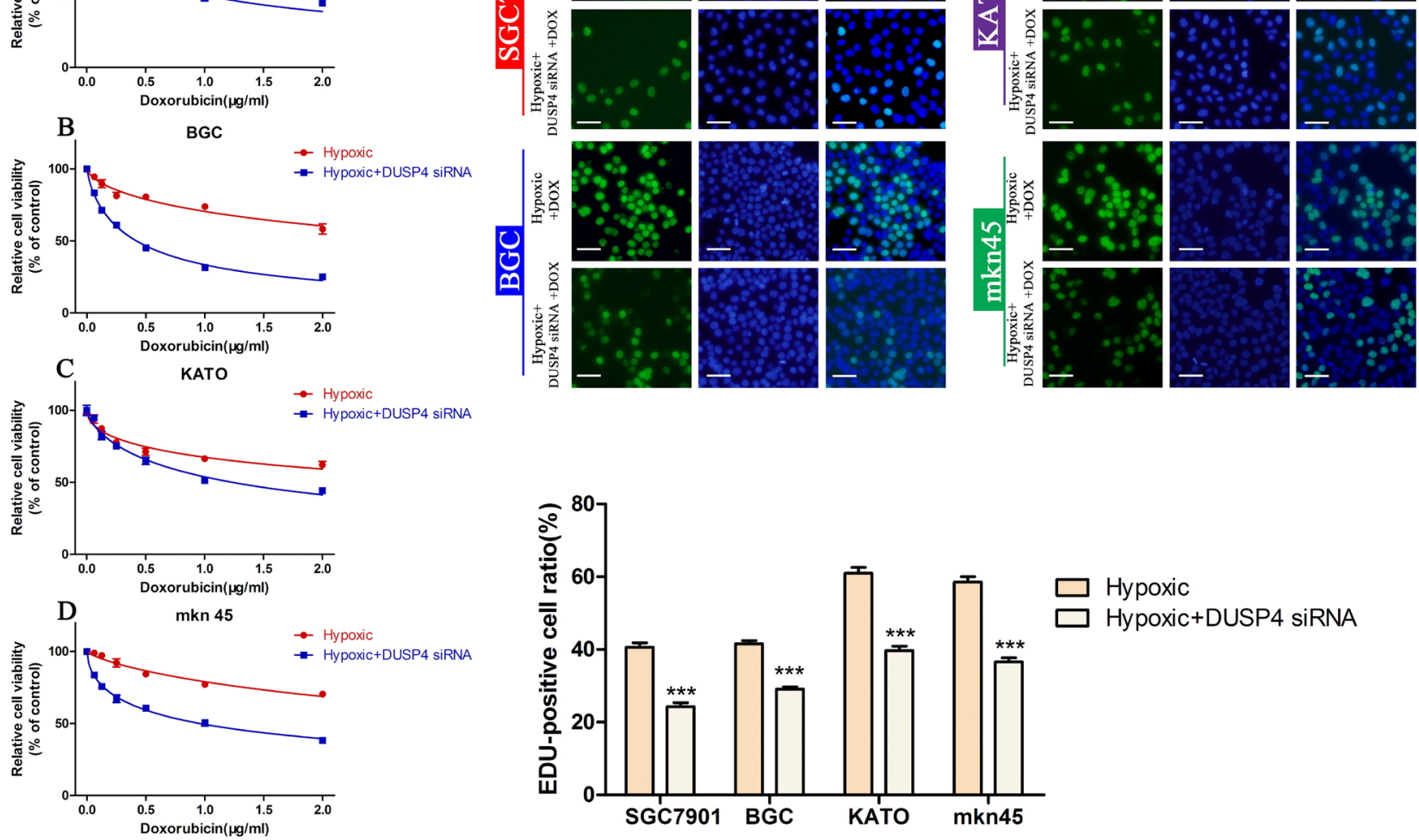

Figure 7: Knocking down DUSP4 reverses hypoxia-induced DOX resistance. (A-D) CCK-8 assay of the viability of GC cells exposed to hypoxia alone or hypoxia + DUSP4 siRNA treated with different concentrations of DOX. (E) EDU assay of the proliferation of $\mathrm{GC}$ cells exposed to hypoxia alone or hypoxia + DUSP4 siRNA treated with their $\mathrm{IC}_{50}$ of DOX $\left({ }^{* * *} P<0.001\right)$. 
Table 6: The viability of GC cells exposed to hypoxia alone or hypoxia + DUSP4 siRNA treated with different concentrations of DOX

\begin{tabular}{lcc}
\hline \multirow{2}{*}{ cell lines } & \multicolumn{2}{c}{$\mathbf{I C}_{50}(\boldsymbol{\mu g} / \mathbf{m l})$} \\
\cline { 2 - 3 } & Hypoxic+DOX & Hypoxic+DUSP4 siRNA+DOX \\
\hline SGC7901 & $2.503(1.544$ to 3.463$)$ & $1.005(0.7426$ to 1.268$)$ \\
BGC & $3.865(2.433$ to 5.297$)$ & $0.4199(0.3964$ to 0.4435$)$ \\
KATO & $4.156(2.366$ to 5.946$)$ & $1.236(1.022$ to 1.450$)$ \\
mkn45 & $5.535(3.560$ to 7.510$)$ & $0.9583(0.8292$ to 1.087$)$ \\
\hline
\end{tabular}

$\mathrm{IC}_{50}$ values show Doxorubicin concentration $[\mu \mathrm{g} / \mathrm{ml}$. mean $(95 \%$ confidence intervals $)]$.

In conclusion, our data demonstrates that high DUSP4 expression may promote the EMT in GC, whereas low DUSP4 expression may enhance DOX sensitivity. However, further research is required to determine the mechanism(s) by which down-regulation of DUSP4 inhibits the EMT and decreases the chemosensitivity of GC cells. Furthermore, these findings indicate that DUSP4 may have potential as a biomarker for monitoring DOX resistance in patients with $\mathrm{GC}$ and could represent a novel treatment target for GC.

\section{MATERIALS AND METHODS}

\section{Cell culture}

BGC-823, KATO III, SGC7901 and MKN45 human gastric cancer cells were purchased from the American Type Culture Collection Cell Biology Collection (ATCC, Manassas, VA, USA). BGC-823, SGC7901 and MKN45 cells were maintained in RPMI-1640 (Gibco, Grand Island, NY, USA); KATO III cells, in DMEM-High glucose (Gibco); all culture media were supplemented with $10 \%$ fetal bovine serum (FBS) and cells were cultured at $37^{\circ} \mathrm{C}$ in a humidified atmosphere of $5 \% \mathrm{CO}_{2}$. Hypoxic cultures were incubated at $37^{\circ} \mathrm{C}$ in a humidified atmosphere of $5 \%$ $\mathrm{CO}_{2}$ and $1 \% \mathrm{O}_{2}$. Doxorubicin was obtained from Sigma (St. Louis, MO, USA) and dissolved in ddH2O.

\section{Cell transfection and RNA interference}

GC cells were transfected with DUSP4 siRNA (100 nmol/L; Santa Cruz Biotechnology, Santa Cruz, CA, USA) or Twist siRNA (100 nmol/L; Santa Cruz) using Lipofectamine 2000 (Invitrogen, Carlsbad, CA, USA) according to the manufacturer's instructions. Non-specific negative control siRNA was purchased from GeneChem Co., Ltd. (Shanghai, China). Six hours after the cells were transfected, the media was replaced with fresh culture media. All assays were performed $48 \mathrm{~h}$ after transfection and repeated three times.

\section{DUSP4 overexpressing plasmid}

The DUSP4 plasmid was designed and constructed by GeneChem Co., Ltd. using the Trans-OE expression vector. The DUSP4 plasmid or control vector (1 $\mu \mathrm{g}$ each) were transfected into GC cells using Lipofectamine 2000 (Invitrogen) according to the manufacturer's instructions; cells were assayed at $48 \mathrm{~h}$ post-transfection.

\section{CCK-8 assay}

Cell viability was assessed using the CCK- 8 assay (Dojindo, Kumamoto, Japan). Approximately 5000 cells were seeded in 96-well plates in $100 \mu \mathrm{L}$ medium. The cells were cultured in media containing 1\% FBS for 24 $\mathrm{h}$ for synchronization, then different concentrations of DOX were added and the cells were incubated for $48 \mathrm{~h}$. CCK-8 solution $(10 \mu \mathrm{l})$ was added to each well, the cells were incubated for $2 \mathrm{~h}$ in the dark, then absorbance was measured at $450 \mathrm{~nm}$ using a MRX II microplate reader (Dynex, Chantilly, VA, USA). We used Prism 5 software (GraphPad, San Diego, CA, USA) to calculate the value of $\mathrm{IC}_{50}$.

\section{Western blotting}

Cells were homogenized in Lysis Buffer (Cell Signaling Technology, Danvers, MA, USA), the supernatants were collected and total protein content was determined using the BCA assay (Thermo Fisher Scientific, Rockford, IL, USA). Equal amounts of protein (40 $\mu \mathrm{g} / \mathrm{lane})$ were separated on SDS-PAGE gels then transferred to $0.22 \mu \mathrm{m}$ PVDF membranes (Millipore, Billerica, MA, USA). Membranes were blocked with 5\% skimmed milk powder in PBST [0.1\% Tween in PBS], incubated with E-cadherin, vimentin, $\beta$-actin or DUSP4 primary antibodies $(1: 1000$; Abcam, Cambridge, MA, USA) overnight at $4^{\circ} \mathrm{C}$, washed five times with PBST for 5 min, incubated with corresponding secondary antibodies (1:2000; abcam) for $1 \mathrm{~h}$ at room temperature, and target bands were visualized using the ECL kit (Millipore). 


\section{EDU assay}

Proliferation was determined using the Click-iTEdU Imaging Kit (Invitrogen) according to the manufacturer's protocol. Briefly, cells were incubated with their $\mathrm{IC}_{50}$ of DOX for $24 \mathrm{~h}$, fixed in $3.7 \%$ formaldehyde for $15 \mathrm{~min}$ at room temperature, and permeabilized in $0.5 \%$ Triton $\mathrm{X}-100$ for $20 \mathrm{~min}$ at room temperature, washed twice with PBS containing 3\% BSA, incubated with $0.5 \mathrm{~mL}$ of Click$\mathrm{iT}^{\circledR}$ reaction cocktail for $30 \mathrm{~min}$ in the dark, then nuclei were counterstained with $1 \mathrm{~mL}$ of $1 \times$ Hoechst 33,342 (1:2000) for $30 \mathrm{~min}$. The numbers of proliferative cells (EDU-positive) in three random fields of view per slide were counted under a fluorescence microscope (Olympus, Tokyo, Japan).

\section{Statistical analysis}

SPSS17.0 software was used for statistical analysis. Data are expressed as the mean $\pm \mathrm{SD}$ of three independent experiments and two groups were compared using the two-tailed Student's $t$-test and one-way analysis of variance (ANOVA) was used to compare multiple groups. Statistical significance was accepted if $P<0.05$.

\section{CONFLICTS OF INTEREST}

The authors have no conflicts of interest to declare.

\section{FUNDING}

This work was sponsored by the National Science Foundation for Young Scholars of China [Grant number 81501380], the National Science Foundation of China [Grant number 81372364], the key research plan and social development project of Jiangsu Province, China [Grant number BE2016603], the National ministry of science and technology projects [Grant number 2016YFC0104105], the Natural Science Foundation for Young Scholars of Jiangsu Province, China [Grant number BK20150110].

\section{REFERENCES}

1. Torre LA, Bray F, Siegel RL, Ferlay J, Lortet-Tieulent J, Jemal A. Global cancer statistics, 2012. CA Cancer J Clin. 2015; 65: 87-108. https://doi.org10.3322/caac.21262.

2. Macdonald JS. Chemotherapy in the management of gastric cancer. J Clin Oncol. 2003; 21: 276s-9s. https://doi. org10.1200/JCO.2003.09.137.

3. De Vivo R, Pignata S, Palaia R, Parisi V, Daniele B. The role of chemotherapy in the management of gastric cancer. J Clin Gastroenterol. 2000; 30: 364-71.

4. Quadri HS, Hong YK, Al-Refaie WB. Approach to the surgical management of resectable gastric cancer. Clin Adv Hematol Oncol. 2016; 14: 249-57.
5. Obermannova R, Lordick F. Management of Metastatic Gastric Cancer. Hematol Oncol Clin North Am. 2017; 31 : 469-83. https://doi.org10.1016/j.hoc.2017.01.006.

6. Irino T, Takeuchi H, Terashima M, Wakai T, Kitagawa Y. Gastric Cancer in Asia: Unique Features and Management. Am Soc Clin Oncol Educ Book. 2017; 37: 279-91. https:// doi.org10.14694/EDBK_175228.

7. MacDonald JS, Schein PS, Woolley PV, Smythe T, Ueno W, Hoth D, Smith F, Boiron M, Gisselbrecht C, Brunet R, Lagarde C. 5-Fluorouracil, doxorubicin, and mitomycin (FAM) combination chemotherapy for advanced gastric cancer. Ann Intern Med. 1980; 93: 533-6.

8. Shibue T, Weinberg RA. EMT, CSCs, and drug resistance: the mechanistic link and clinical implications. Nat Rev Clin Oncol. 2017. https://doi.org10.1038/nrclinonc.2017.44.

9. Mitra A, Mishra L, Li S. EMT, CTCs and CSCs in tumor relapse and drug-resistance. Oncotarget. 2015; 6: 10697 711. https://doi.org10.18632/oncotarget.4037.

10. Hu SH, Wang CH, Huang ZJ, Liu F, Xu CW, Li XL, Chen GQ. miR-760 mediates chemoresistance through inhibition of epithelial mesenchymal transition in breast cancer cells. Eur Rev Med Pharmacol Sci. 2016; 20: 5002-8.

11. Sadreddini S, Safaralizadeh R, Baradaran B, AghebatiMaleki L, Hosseinpour-Feizi MA, Shanehbandi D, Jadidi-Niaragh F, Sadreddini S, Kafil HS, Younesi V, Yousefi M. Chitosan nanoparticles as a dual drug/siRNA delivery system for treatment of colorectal cancer. Immunol Lett. 2017; 181: 79-86. https://doi.org10.1016/j. imlet.2016.11.013.

12. Kubiliute R, Sulskyte I, Daniunaite K, Daugelavicius R, Jarmalaite $\mathrm{S}$. Molecular features of doxorubicin-resistance development in colorectal cancer CX-1 cell line. Medicina (Kaunas). 2016; 52: 298-306. https://doi.org10.1016/j. medici.2016.09.003.

13. Kohnoh T, Hashimoto N, Ando A, Sakamoto K, Miyazaki S, Aoyama D, Kusunose M, Kimura M, Omote N, Imaizumi K, Kawabe T, Hasegawa Y. Hypoxia-induced modulation of PTEN activity and EMT phenotypes in lung cancers. Cancer Cell Int. 2016; 16: 33. https://doi.org10.1186/ s12935-016-0308-3.

14. Fang S, Wu L, Li M, Yi H, Gao G, Sheng Z, Gong P, Ma Y, Cai L. ZEB1 knockdown mediated using polypeptide cationic micelles inhibits metastasis and effects sensitization to a chemotherapeutic drug for cancer therapy. Nanoscale. 2014; 6: 10084-94. https://doi.org10.1039/c4nr01518e.

15. Du B, Shim JS. Targeting Epithelial-Mesenchymal Transition (EMT) to Overcome Drug Resistance in Cancer. Molecules. 2016; 21. https://doi.org10.3390/ molecules21070965.

16. Han R, Xiong J, Xiao R, Altaf E, Wang J, Liu Y, Xu H, Ding $\mathrm{Q}$, Zhang Q. Activation of beta-catenin signaling is critical for doxorubicin-induced epithelial-mesenchymal transition in BGC-823 gastric cancer cell line. Tumour Biol. 2013; 34 : 277-84. https://doi.org10.1007/s13277-012-0548-3. 
17. Han RF, Ji X, Dong XG, Xiao RJ, Liu YP, Xiong J, Zhang QP. An epigenetic mechanism underlying doxorubicin induced EMT in the human BGC-823 gastric cancer cell. Asian Pac J Cancer Prev. 2014; 15: 4271-4.

18. Low HB, Zhang Y. Regulatory Roles of MAPK Phosphatases in Cancer. Immune Netw. 2016; 16: 85-98. https://doi.org10.4110/in.2016.16.2.85.

19. Saigusa S, Inoue $\mathrm{Y}$, Tanaka K, Toiyama Y, Okugawa Y, Shimura T, Hiro J, Uchida K, Mohri Y, Kusunoki M. Decreased expression of DUSP4 is associated with liver and lung metastases in colorectal cancer. Med Oncol. 2013; 30: 620. https://doi.org10.1007/s12032-013-0620-x.

20. Jung HH, Lee SH, Kim JY, Ahn JS, Park YH, Im YH. Statins affect ETS1-overexpressing triple-negative breast cancer cells by restoring DUSP4 deficiency. Sci Rep. 2016; 6: 33035. https://doi.org10.1038/srep33035.

21. Hijiya N, Tsukamoto Y, Nakada C, Tung Nguyen L, Kai T, Matsuura K, Shibata K, Inomata M, Uchida T, Tokunaga A, Amada K, Shirao K, Yamada Y, et al. Genomic Loss of DUSP4 Contributes to the Progression of Intraepithelial Neoplasm of Pancreas to Invasive Carcinoma. Cancer Res. 2016; 76: 2612-25. https://doi.org 10.1158/0008-5472. CAN-15-1846.

22. Schmid CA, Robinson MD, Scheifinger NA, Muller S, Cogliatti S, Tzankov A, Muller A. DUSP4 deficiency caused by promoter hypermethylation drives JNK signaling and tumor cell survival in diffuse large B cell lymphoma. J Exp Med. 2015; 212: 775-92. https://doi.org10.1084/ jem.20141957.

23. Hasegawa T, Enomoto A, Kato T, Kawai K, Miyamoto R, Jijiwa M, Ichihara M, Ishida M, Asai N, Murakumo Y, Ohara K, Niwa Y, Goto H, et al. Roles of induced expression of MAPK phosphatase-2 in tumor development in RET-MEN2A transgenic mice. Oncogene. 2008; 27: 5684-95. https://doi.org10.1038/onc.2008.182.

24. Lawan A, Al-Harthi S, Cadalbert L, McCluskey AG, Shweash M, Grassia G, Grant A, Boyd M, Currie S, Plevin R. Deletion of the dual specific phosphatase-4 (DUSP4) gene reveals an essential non-redundant role for MAP kinase phosphatase-2 (MKP-2) in proliferation and cell survival. J Biol Chem. 2011; 286: 12933-43. https://doi. org10.1074/jbc.M110.181370.

25. Kim H, Jang SM, Ahn H, Sim J, Yi K, Chung Y, Han H, Rehman A, Chung MS, Jang K, Paik SS. Clinicopathological significance of dual-specificity protein phosphatase 4 expression in invasive ductal carcinoma of the breast. J Breast Cancer. 2015; 18: 1-7. https://doi. $\operatorname{org} 10.4048 /$ jbc.2015.18.1.1.

26. Lasham A, Mehta SY, Fitzgerald SJ, Woolley AG, Hearn JI, Hurley DG, Ruza I, Algie M, Shelling AN, Braithwaite AW, Print CG. A novel EGR-1 dependent mechanism for YB-1 modulation of paclitaxel response in a triple negative breast cancer cell line. Int J Cancer. 2016; 139: 1157-70. https:// doi.org10.1002/ijc.30137.
27. Cushman SM, Jiang C, Hatch AJ, Shterev I, Sibley AB, Niedzwiecki D, Venook AP, Owzar K, Hurwitz HI, Nixon AB. Gene expression markers of efficacy and resistance to cetuximab treatment in metastatic colorectal cancer: results from CALGB 80203 (Alliance). Clin Cancer Res. 2015; 21: 1078-86. https://doi.org10.1158/1078-0432.CCR-14-2313.

28. Liu Y, Du F, Chen W, Yao M, Lv K, Fu P. Knockdown of dual specificity phosphatase 4 enhances the chemosensitivity of MCF-7 and MCF-7/ADR breast cancer cells to doxorubicin. Exp Cell Res. 2013; 319: 3140-9. https://doi.org10.1016/j.yexcr.2013.08.023.

29. Rottenberg S, Jonkers J. MEK inhibition as a strategy for targeting residual breast cancer cells with low DUSP4 expression. Breast Cancer Res. 2012; 14: 324. https://doi. org10.1186/bcr3327.

30. Balko JM, Cook RS, Vaught DB, Kuba MG, Miller TW, Bhola NE, Sanders ME, Granja-Ingram NM, Smith JJ, Meszoely IM, Salter J, Dowsett M, Stemke-Hale K, et al. Profiling of residual breast cancers after neoadjuvant chemotherapy identifies DUSP4 deficiency as a mechanism of drug resistance. Nat Med. 2012; 18: 1052-9. https://doi. org 10.1038/nm.2795.

31. Tang H, Massi D, Hemmings BA, Mandala M, Hu Z, Wicki A, Xue G. AKT-ions with a TWIST between EMT and MET. Oncotarget. 2016; 7: 62767-77. https://doi. org10.18632/oncotarget.11232.

32. Zuo J, Wen J, Lei M, Wen M, Li S, Lv X, Luo Z, Wen G. Hypoxia promotes the invasion and metastasis of laryngeal cancer cells via EMT. Med Oncol. 2016; 33: 15. https://doi. org10.1007/s12032-015-0716-6.

33. Misra A, Pandey C, Sze SK, Thanabalu T. Hypoxia activated EGFR signaling induces epithelial to mesenchymal transition (EMT). PLoS One. 2012; 7: e49766. https://doi. org10.1371/journal.pone.0049766.

34. Wang X, Pang L, Feng J. A phase II study of etoposide, doxorubicin, and carboplatin in the treatment of advanced gastric cancer. Am J Clin Oncol. 2002; 25: 71-5.

35. Baglia ML, Cai Q, Zheng Y, Wu J, Su Y, Ye F, Bao PP, Cai H, Zhao Z, Balko J, Zheng W, Lu W, Shu XO. Dual specificity phosphatase 4 gene expression in association with triple-negative breast cancer outcome. Breast Cancer Res Treat. 2014; 148: 211-20. https://doi.org10.1007/ s10549-014-3127-z.

36. De Vriendt V, De Roock W, Di Narzo AF, Tian S, Biesmans B, Jacobs B, Budinska E, Sagaert X, Rossi S, D'Ario G, Delorenzi M, Simon I, Vecchione L, et al. DUSP 4 expression identifies a subset of colorectal cancer tumors that differ in MAPK activation, regardless of the genotype. Biomarkers. 2013; 18: 516-24. https://doi.org10.3109/1354 750X.2013.819038.

37. Groschl B, Bettstetter M, Giedl C, Woenckhaus M, Edmonston T, Hofstadter F, Dietmaier W. Expression of the MAP kinase phosphatase DUSP4 is associated with microsatellite instability in colorectal cancer (CRC) and 
causes increased cell proliferation. Int J Cancer. 2013; 132: 1537-46. https://doi.org10.1002/ijc.27834.

38. Sim J, Yi K, Kim H, Ahn H, Chung Y, Rehman A, Jang SM, Lee KH, Jang K, Paik SS. Immunohistochemical expression of dual-specificity protein phosphatase 4 in patients with colorectal adenocarcinoma. Gastroenterol Res Pract. 2015; 2015: 283764. https://doi.org10.1155/2015/283764.

39. Zhang R, Wang G, Zhang PF, Zhang J, Huang YX, Lu YM, Da W, Sun Q, Zhu JS. Sanguinarine inhibits growth and invasion of gastric cancer cells via regulation of the DUSP4/ERK pathway. J Cell Mol Med. 2017; 21: 1117-27. https://doi.org10.1111/jcmm.13043.

40. Singh A, Settleman J. EMT, cancer stem cells and drug resistance: an emerging axis of evil in the war on cancer.
Oncogene. 2010; 29: 4741-51. https://doi.org10.1038/ onc.2010.215.

41. Xu J, Liu D, Niu H, Zhu G, Xu Y, Ye D, Li J, Zhang Q. Resveratrol reverses Doxorubicin resistance by inhibiting epithelial-mesenchymal transition (EMT) through modulating PTEN/Akt signaling pathway in gastric cancer. J Exp Clin Cancer Res. 2017; 36: 19. https://doi.org10.1186/ s13046-016-0487-8.

42. Boulding $\mathrm{T}, \mathrm{Wu} F$, McCuaig R, Dunn J, Sutton CR, Hardy K, Tu W, Bullman A, Yip D, Dahlstrom JE, Rao S. Differential Roles for DUSP Family Members in Epithelial-to-Mesenchymal Transition and Cancer Stem Cell Regulation in Breast Cancer. PLoS One. 2016; 11: e0148065. https://doi.org10.1371/journal.pone.0148065. 